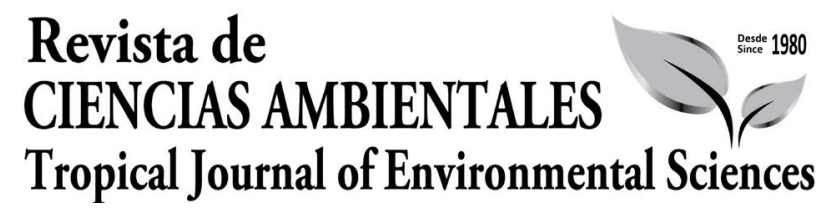

Revista de Ciencias Ambientales (Trop J Environ Sci) e-ISSN: 2215-3896 (Enero-Junio, 2022) . Vol 56(1): 259-267 DOI: https://doi.org/10.15359/rca.56-1.13 Open Access: www.revistas.una.ac.cr/ambientales e-mail: revista.ambientales@una.ac.cr Chaves A..

\title{
BIOGRAFÍA \\ Peter Pritchard: ciudadano del mundo, investigador y protector de las tortugas
}

\author{
Peter Pritchard: citizen of the world, researcher and protector of turtles
}

\author{
Anny Chaves Quirós ${ }^{1}$
}

\section{Introducción}

Las tortugas del mundo, en todo lo amplio que este concepto pueda abarcar, fueron el interés de estudio de Peter Pritchard. Procuró conocer cada especie en detalle, de preferencia ver con sus propios ojos un espécimen vivo o alguna parte de su osamenta y, en última instancia, una fotografía. Después, aprovecharía la primera oportunidad para desplazarse a cualquier recóndito lugar del planeta, para tener ese contacto directo con la especie.

Maravillado desde niño por este grupo de reptiles, dedicó su vida a la conservación y estudio de todos los grupos de tortugas, así como a entender los aspectos que permitieron que las tortugas lograran sobrevivir el período de transición entre el Cretácico y Paleoceno, cuando la mayoría de los reptiles se extinguieron. Desde su punto de vista, esos animales únicos, metidos dentro de un caparazón, que misteriosamente se han adaptado a un mundo cambiante y todavía están con nosotros, merecían toda su atención y esfuerzo para conservarlos (Pritchard, 1967).

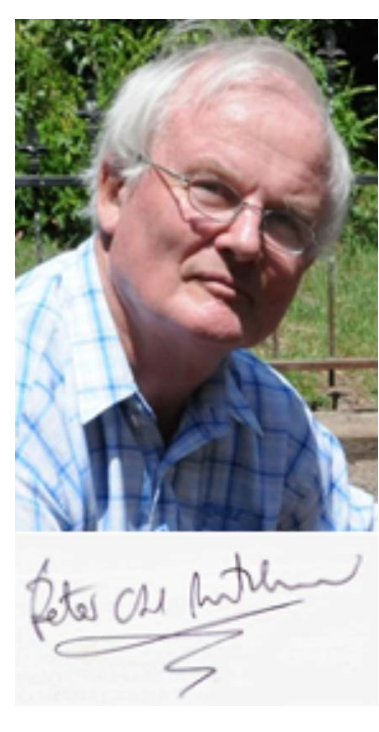

Peter tuvo muy claro siempre que la conservación de las especies iba más allá de conocerlas. Era necesario divulgar su importancia, entender las culturas que las utilizan, encontrar las opciones para un uso sostenible y cuando no era posible, abogaba por drásticas medidas de conservación. Fue así como estuvo involucrado en un gran número de organizaciones tanto nacionales como internacionales, aportando su esfuerzo y trabajo para obtener logros concretos: sitios protegidos, declaraciones fuertes en contra del abuso comercial de especies, documentos científicos que justificaban acciones urgentes en favor de las tortugas y mucha información que permitió conocer mejor a esos increíbles animales.

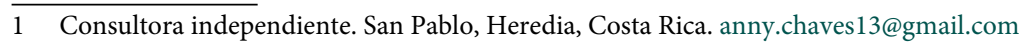

\begin{tabular}{|c|c|c|}
\hline 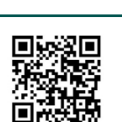 & (c) (1) () () (2) & 259 \\
\hline
\end{tabular}




\section{Revista de CIENCIAS AMBIENTALES Tropical Journal of Environmental Sciences}

Revista de Ciencias Ambientales (Trop J Environ Sci)

e-ISSN: 2215-3896

(Enero-Junio, 2022) . Vol 56(1): 259-267

DOI: https://doi.org/10.15359/rca.56-1.13

Open Access: www.revistas.una.ac.cr/ambientales e-mail: revista.ambientales@una.ac.cr Chaves A..

\section{Sus orígenes y su vida familiar}

Peter Charles Howard Pritchard nació el 23 de junio de 1943, en Hertfordshire, Inglaterra. Cuando tenía 9 años, se trasladaron a Belfast, Irlanda, ya que su padre fue trasladado para ser profesor de Anatomía en Queen's University.

Sus primeros estudios los realizó en el Campbell College, en Belfast, donde se interesó por la Física. Fue admitido en el Magdalen College de la Universidad de Oxford, en 1961, como estudiante de Química. Participó como investigador en el laboratorio de Bioquímica, en donde su interés por la Biología se fue acrecentando (Pritchard, 1967).

Su fascinación por las tortugas había nacido desde que visitó de niño, con sus padres, el zoológico de Londres. Al identificar el interés de su hijo por ese animal, sus padres le compraron una tortuga griega y, desde entonces, fueron su compañía. Durante sus días de estudiante siempre mantuvo un terrario con tortugas en su dormitorio (Fretey, 2021).

Se graduó con honores en Oxford en 1964. Ese mismo año fue hospitalizado por una grave enfermedad y cuando se recuperó aprovechó la invitación del Gobernador de Guiana Británica (ahora Guyana), quien era amigo de su padre, para realizar una expedición a Sur América (Fretey, 2021). En su recorrido por la costa, topó un día con una horrorosa imagen: varias docenas de tortugas baula destazadas en la playa. Tan pronto como pudo, escribió al príncipe Philip de Edimburgo, quien era el presidente de WWF-Reino Unido, denunciado la situación y solicitando acciones. La carta llegó a manos de Archie F. Carr (considerado la autoridad líder mundial en tortugas marinas), en la Universidad de Florida, quien quedó impresionado por la calidad de las observaciones del joven Pritchard (Fretey, 2021). En 1965, fue admitido como estudiante de doctorado en la Universidad de Florida (Gainesville) bajo la tutoría de Carr, donde elaboró su tesis de doctorado sobre la tortuga lora (Lepidochelys olivacea), realizando estudios de campo en la Guiana Holandesa (ahora Suriname) con el investigador holandés Joop Schultz (Fretey, 2021).

En uno de sus viajes a Guyana, Peter conoció a la periodista Sibille Hart, una mujer pequeña, de piel bronceada, con un carácter fuerte capaz de encantar al gran investigador. Se casaron y establecieron su residencia en un pequeño pueblo cerca de Orlando, Florida, llamado Oviedo. Ellos procrearon tres varones (Dominic, Sebastian y Cameron), de los cuales el mayor se enlistó en el ejército y falleció en la guerra del Golfo Pérsico, lo que significó un duro golpe para la familia.

\section{Sus aportes profesionales}

Desde estudiante, empezó a escribir, no solo publicaciones de artículos científicos, sino también material de divulgación popular. En su primer libro Living turtles of the world (Las tortugas vivas del mundo), publicado en 1967, señala precisamente la necesidad de cerrar la brecha entre una publicación científica y una revista de aficionados o de coleccionistas, donde con un lenguaje sencillo se pueda explicar ampliamente la biología del grupo. Esta manera de identificar la importancia de llevar la ciencia a un público mayor lo va a caracterizar en su monumental obra científica. Este libro fue ampliado posteriormente por La Enciclopedia de las Tortugas

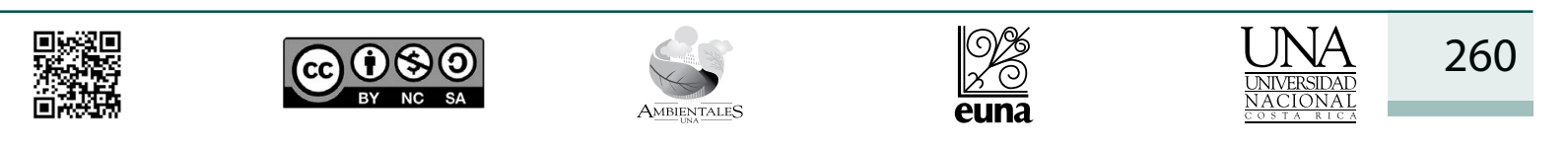




\section{Revista de CIENCIAS AMBIENTALES Tropical Journal of Environmental Sciences}

Revista de Ciencias Ambientales (Trop J Environ Sci)

e-ISSN: 2215-3896

(Enero-Junio, 2022) . Vol 56(1): 259-267

DOI: https://doi.org/10.15359/rca.56-1.13

Open Access: www.revistas.una.ac.cr/ambientales e-mail: revista.ambientales@una.ac.cr Chaves A..

(Pritchard, 1979), en la misma línea de proveer la mejor información científica de manera accesible al público y llegó a convertirse en la "Biblia" de las Tortugas. Cientos de publicaciones en revistas científicas y una serie de sinopsis de especies destacan en su producción científica, además de trabajos en coautoría con un gran número de investigadores.

En una reciente recopilación de su obra científica, Rhodin y Thompson (2020) reunieron 215 artículos, aunque confiesan que no es la lista completa (ya que Peter no llevaba su propia lista de publicaciones), esperan ir completando, mientras siguen revisando revistas y otras publicaciones.

Intervino en las más diversas polémicas mundiales sobre la explotación y conservación de recursos naturales. Buscaba las alternativas sostenibles, mediando entre derechos culturales y la protección de una especie, pero, si el peligro era inminente para la especie, levantaba con fuerza la bandera para mover cielo y tierra, para buscar su protección. Su criterio tenía un valor por sí mismo.

Le entusiasmaba participar en las reuniones anuales sobre tortugas marinas, que se realizaban primero en la costa este de los Estados Unidos y después en diferentes partes del mundo, ya organizadas como un gran simposio con la asistencia de más 500 personas. En ellas circulaba por los diferentes grupos regionales, procuraba compartir y dedicar tiempo a las sesiones de trabajo de los jóvenes investigadores, tanto las académicas como las sociales. Identificaba las futuras estrellas y de alguna manera gestionaba apoyo. Peter tenía un carácter jovial, muy buen humor y se puede señalar que hasta pícaro. Calzaba muy bien en el grupo latino, participaba con nosotros en los tiempos extra y gozamos siempre de su presencia, aporte y protección.

Realizó investigaciones de tortugas marinas en México, Costa Rica, Venezuela, Guyana, Surinam, y en una inmensa lista de países de todos los continentes. Su principal interés era descubrir nuevos sitios importantes para las diferentes etapas de la vida de las tortugas marinas, presentarlos ante el mundo científico y buscar las opciones para su conservación.

Muchas de sus publicaciones están orientadas a aclarar varios de los problemas taxonómicos de los diferentes géneros y especies de tortugas, así como la descripción de nuevas especies.

En una de las reuniones sobre tortugas marinas, en la sobremesa durante una cena, discutía con varios colegas sobre la relación de las especies de Lepidochelys y cómo había sido la colonización y distribución de la L. olivacea en todos los océanos del planeta. Peter había propuesto la hipótesis, desde 1969, de que L. kempi y L. olivacea se separaron ancestralmente. La población inicial del Atlántico había sido dividida al cerrarse el istmo de Centroamérica, quedando L. kempi restringida al Golfo de México, y L. olivacea en el Pacífico de América, desde donde colonizó después el Pacífico de Asia, el Índico y vía Sur África colonizó el Atlántico africano y la costa Atlántica de Sur América. Mientras, el resto de los participantes de la discusión proponían, que, si bien se habían separado las dos especies al emerger el istmo, una población de $L$. olivacea había quedado en Sur América y fue la que colonizó el Atlántico de África, el Índico y el Pacífico de Asia. Años después, con el avance de la genética, en un estudio colaborativo, donde

\begin{tabular}{|c|c|c|c|c|c|}
\hline 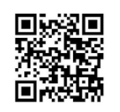 & (c) (1) (5) () & $\overbrace{\text { AMBIENTALS }}$ & $\frac{O \%}{20 \%}$ & 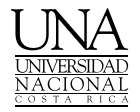 & 261 \\
\hline
\end{tabular}




\section{Revista de CIENCIAS AMBIENTALES Tropical Journal of Environmental Sciences}

Revista de Ciencias Ambientales (Trop J Environ Sci)

e-ISSN: 2215-3896

(Enero-Junio, 2022) . Vol 56(1): 259-267

DOI: https://doi.org/10.15359/rca.56-1.13

Open Access: www.revistas.una.ac.cr/ambientales e-mail: revista.ambientales@una.ac.cr Chaves A..

muchos investigadores aportamos muestras y trabajo para terminar de dilucidar esta discusión, dio como resultado contundente el apoyo a la hipótesis de Peter (Bowen et al., 1998).

Como expedicionario y trotamundos, realizó muchos viajes en busca de identificar particularidades, como reconocer nuevos sitios de anidación de tortugas marinas, rescatar especies perdidas o describir nuevas especies. Por un solo rumor de alguna situación que le resultara especial, buscaba los medios y el financiamiento para lograr su quimera.

Fue así como logró rescatar al último espécimen de la tortuga galápagos de la Isla Pinta, en el Archipiélago de Galápagos, a partir de una foto que le enseñó el malacólogo húngaro József Vágvölgyi, de una tortuga gigante que parecía tener una silla de montar en su espalda. Peter participó en una expedición a Isla Pinta en 1972, donde encontraron un espécimen de esa tortuga y lo llevaron a la Estación Charles Darwin, en la Isla Santa Cruz, donde lo cuidaron y lo llamaron el solitario George y fue confirmado como el último de la especie Chelonoidis abingdonii (Günther, 1877). Peter organizó seis expediciones a Galápagos, tratando de encontrar otro espécimen, sin éxito. En el programa de reproducción de tortugas galápagos trataron de cruzarlo con especies cercanas sin lograr reproducirlo; el solitario George murió en 2012 (Fretey, 2021).

\section{Peter y Costa Rica}

En 1982, el simposio WATS I (Western Atlantic Turtle Symposium) se celebró en Costa Rica. Todos los autores que estábamos en el curso de tortugas marinas en la Escuela de Biología de la UCR, nos encontrábamos reunidos en el Hotel Costa Rica. Gracias al privilegio de ser estudiantes del herpetólogo Douglas C. Robinson, en los recesos y en las sesiones de trabajo, nos fueron presentando con los grandes "tortugólogos" del mundo, encabezando la lista Archie F. Carr, Peter Pritchard, Jacques Fretey, Colin Limpus, Fred Berry, Ross Witham, Karen Bjorndall, Jack Woody, George Balazs, Jim Richardson y muchos más. Era como estar con estrellas de cine para nosotros, la mayoría de ellos muy accesibles y dispuestos a responder preguntas.

Por su personalidad y figura, Peter destacaba en donde estuviera. La primera vez que lo vi, vestía una chamarra kaki, como estilo safari. Parecía exactamente un explorador inglés del siglo XIX. Su cabeza sobresalía por encima de todos los representantes de las diferentes delegaciones, su acento en inglés era muy particular, no solo por lo británico, sino también las trazas de irlandés y norteamericano. Hablaba francés y español con bastante fluidez, lo que facilitaba el abordaje de los estudiantes. Nos acercamos a preguntarle sobre qué pensaba de separar la tortuga negra (Chelonia mydas agassizi) como especie diferente a la tortuga verde (Chelonia mydas mydas), un tema de gran discusión en ese tiempo, que también ha tenido respuesta a través de la genética. Peter nos condujo a una sala de reuniones y, con ayuda de un rotafolio, nos dio una clase completa con tres opciones de respuesta. Quedamos estupefactos, no solo por el contenido de sus argumentos, sino por la atención que dio a ese grupo de curiosos estudiantes.

Mi segundo encuentro fue al llegar a una reunión de Widecast en Isla Guadalupe en 1985. Peter y Jacques Fretey me esperaban en el pequeño aeropuerto de Pointe à Pitre, para llevarme al hotel donde se celebraba la reunión. Con una mezcla de español y francés, en medio de

\begin{tabular}{|c|c|c|}
\hline 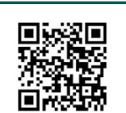 & 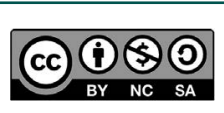 & 262 \\
\hline
\end{tabular}




\section{Revista de CIENCIAS AMBIENTALES Tropical Journal of Environmental Sciences}

Revista de Ciencias Ambientales (Trop J Environ Sci)

e-ISSN: 2215-3896

(Enero-Junio, 2022) . Vol 56(1): 259-267

DOI: https://doi.org/10.15359/rca.56-1.13

Open Access: www.revistas.una.ac.cr/ambientales e-mail: revista.ambientales@una.ac.cr

Chaves A..

discusiones entre ellos dos para ponerse de acuerdo, me fueron describiendo el mapa de actores para que llegara ubicada; ya que, por un contratiempo antes de mi salida de San José, no pude viajar con todo el grupo que se había reunido de previo en Miami.

En esa actividad yo presentaría un plan de acción para las tortugas marinas del Caribe de Costa Rica, donde se planteaban opciones reguladas para el aprovechamiento de las tortugas marinas por las comunidades costeras y actividades alternativas para respaldar la economía. En ese contexto, era un planteamiento complejo, porque la mayoría iban más por el tema de la conservación absoluta, por lo que Peter, Jacques y Ross Withan fueron fundamentales en esas reuniones, para apoyarme y valorar los planteamientos (Chaves, 2021). En esa reunión, fui integrada como un miembro del grupo de "tortugólogos" del mundo, también fue el principio de una fuerte amistad con Peter y Jacques.

Peter había estado por primera vez a Costa Rica para participar como estudiante en Tortuguero en los años 65-70 y siempre encontró un buen motivo para venir. Muchas veces, organizaba expediciones, promovía documentales, reuniones de especialistas o simplemente venía a ver tortugas (Figura 1).

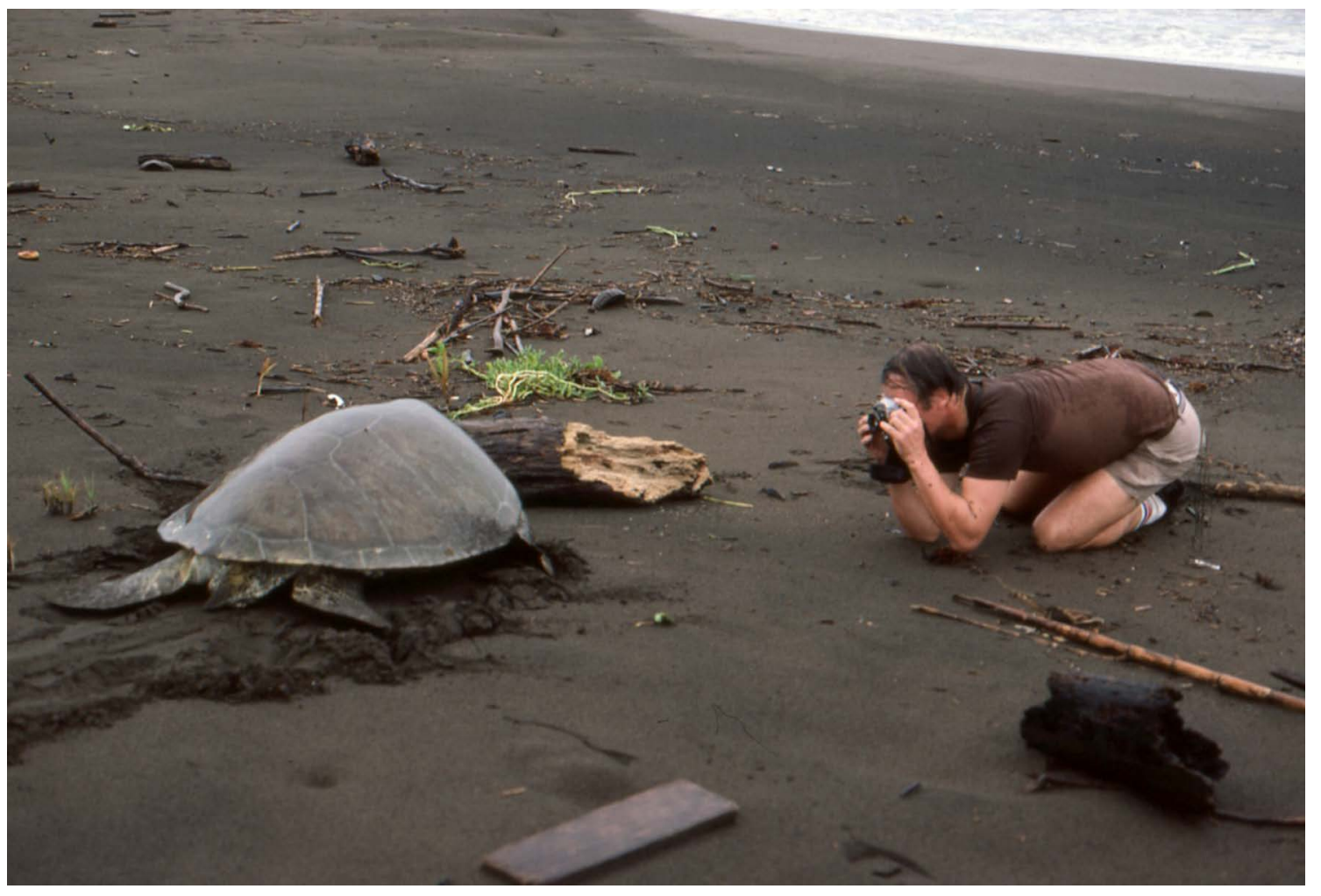

Figura 1. Peter en Tortuguero, Costa Rica, en 1983. Fotografía: Jacques Fretey.

Figure 1. Peter in Tortuguero, Costa Rica, in 1983. Photography: Jacques Fretey.

\begin{tabular}{|c|c|c|}
\hline 品裙品 & (c) (i) (5) (2) & 263 \\
\hline
\end{tabular}




\section{Revista de CIENCIAS AMBIENTALES Tropical Journal of Environmental Sciences}

Revista de Ciencias Ambientales (Trop J Environ Sci)

e-ISSN: 2215-3896

(Enero-Junio, 2022) . Vol 56(1): 259-267

DOI: https://doi.org/10.15359/rca.56-1.13

Open Access: www.revistas.una.ac.cr/ambientales e-mail: revista.ambientales@una.ac.cr Chaves A.

Se aparecía en el país muchas veces sin avisar y con la ventaja de hablar español, se manejaba sin problema por San José y zonas rurales para llegar a cualquier lado. Conversaba con la gente en la calle y se interesaba por detalles de la vida cotidiana de las personas del lugar. Una vez, en ruta hacia Nancite, Parque Nacional Santa Rosa, mientras cargábamos el tanque de combustible, entabló conversación con los niños que venden ocarinas en el cruce de Liberia y terminó almorzando en la casa de esa familia. Al regreso del trabajo en Nancite, nos pidió pasar al súper mercado y llevar un diario a esa familia liberiana.

Entre sus anécdotas, contaba que en 2008 estuvo a punto de morir en Costa Rica, ya que fue atropellado por un vehículo cuando trataba de cruzar la calle entre La Sabana y el Ministerio de Agricultura (Ceriani, 2021), solo por la precisa de llegar antes de que cerraran, al Museo de La Salle, donde quería mostrar a una estudiante, los interesantes especímenes que se custodian en esa colección. Las heridas fueron superficiales y se levantó y siguió su camino al museo, pero cuando llegaron ya el museo estaba cerrado, por lo que al día siguiente volvió para cumplir con su objetivo.

Con su asesoría al gobierno de Costa Rica, se logró consolidar la protección de las playas de anidación de tortugas marinas, en particular la ampliación del Refugio de Vida Silvestre Tamarindo como Parque Nacional Marino Las Baulas. También medió en las diferentes situaciones relacionadas a la captura de tortugas verdes y a la recolección de huevos de la tortuga lora.

Impulsó la imagen de Costa Rica en la protección de las tortugas, donde actuando como asesor científico de documentales, promovió que estos se realizaran en locaciones en el país como en Nancite, Tortuguero, Playa Grande y Ostional. Lo acompañé muchas veces en sus aventuras por el país. Caminamos muchos kilómetros de playa juntos, en los que tuve la gran oportunidad de escuchar sus historias alrededor del mundo, pero sobre todo sus conocimientos.

\section{Su colección de tortugas}

Muchas veces, visité su casa junto con Douglas Robinson. Era parada obligatoria en nuestros viajes, para asistir a las reuniones de tortugas marinas que se celebraban en la costa este de Estados Unidos. No importaba si éramos dos o cinco, igual siempre había hospedaje para todos. Colchones o sacos de dormir al suelo y la sala quedaba convertida en dormitorio.

Peter tenía una gran colección de tortugas vivas que caminaban por el jardín, pero cuando la noche se enfriaba, tenían su puertecilla para ingresar a la casa. Recuerdo despertar una vez con una enorme tortuga terrestre empujándome para acercarse al calentador, lugar que yo había escogido por la misma razón. También tenía una enorme colección de caparazones y osamentas de tortugas, de todas partes del mundo, bien organizados en cajas con su nombre escrito en un papel arrollado y metido dentro del caparazón. La primera vez que me enseñó su colección, la tenía en el ático y me regaló un espécimen de una tortuga suramericana del grupo de las pleurodeiras, que no hay en Costa Rica y que siempre me han atraído.

Esta colección personal, tanto de especímenes vivos como preservados, se convirtió posteriormente en parte del legado que Peter dejó al constituir el Museo del Chelonian Research

\begin{tabular}{|c|c|c|}
\hline 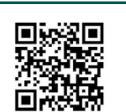 & (c) $\underset{\mathrm{BY}}{(1)}\left(\begin{array}{l}\mathrm{NC} \\
\mathrm{SA}\end{array}\right.$ & 264 \\
\hline
\end{tabular}




\section{Revista de CIENCIAS AMBIENTALES Tropical Journal of Environmental Sciences}

Revista de Ciencias Ambientales (Trop J Environ Sci)

e-ISSN: 2215-3896

(Enero-Junio, 2022) . Vol 56(1): 259-267

DOI: https://doi.org/10.15359/rca.56-1.13

Open Access: www.revistas.una.ac.cr/ambientales e-mail: revista.ambientales@una.ac.cr

Chaves A..

Institute (Figura 2). Este museo está ubicado al frente de la casa de Peter en Oviedo, en ella se encuentra la segunda más completa colección de tortugas de todo el mundo. Cuenta con 16500 especímenes que cubren el $86 \%$ de las especies existentes. La propiedad incluye un espacio de terreno, donde se ubicaron a las tortugas vivas. El Instituto cuenta con una impresionante biblioteca y, además, tiene facilidades para albergar estudiantes que vienen de diferentes partes del mundo a realizar estudios temporales, contribuyendo así a la formación de estos investigadores.

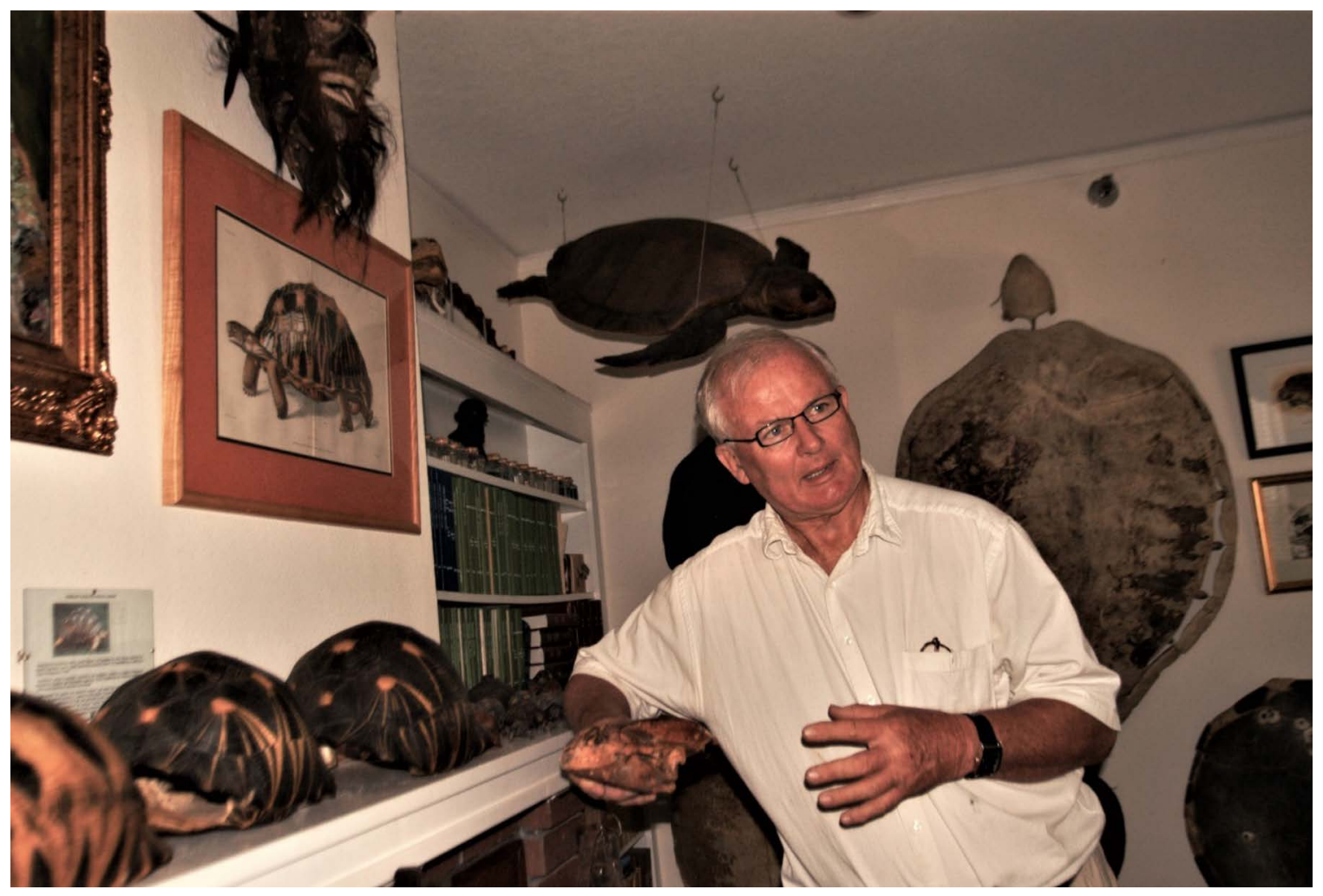

Figura 2. Peter en el Museo del Chelonian Research Institute. Fotografía: Franck Bonin.

Figure 2. Peter in the Museum of the Chelonian Research Institute. Photography: Franck Bonin.

Su preocupación por apoyar la formación de jóvenes y promover el entusiasmo por el conocimiento de las tortugas es otro de los elementos que han caracterizado su personalidad y, por lo tanto, su aporte. Como director de investigaciones de la Florida Audubon Society, promovió un premio de conservación para investigadores de América Latina, del cual recibí el honor en 1985. Peter promovió realizar la ceremonia de entrega de mi premio en Costa Rica. Esta se realizó en el Museo Nacional, con la participación del presidente Oscar Arias Sánchez, la primera dama Margarita Penón y los directores de Chelonian Research Institute Robert y Marie Truland, logrando con esta actividad un importante apoyo a las diferentes iniciativas relacionadas a las tortugas marinas en el país.

\begin{tabular}{|c|c|c|}
\hline 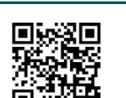 & (c) (i) (\$) (2) & 265 \\
\hline
\end{tabular}




\section{Revista de CIENCIAS AMBIENTALES Tropical Journal of Environmental Sciences}

Revista de Ciencias Ambientales (Trop J Environ Sci)

e-ISSN: 2215-3896

(Enero-Junio, 2022) . Vol 56(1): 259-267

DOI: https://doi.org/10.15359/rca.56-1.13

Open Access: www.revistas.una.ac.cr/ambientales e-mail: revista.ambientales@una.ac.cr Chaves A..

\section{Sus tiempos finales}

Peter fue diagnosticado con Alzheimer, pero aun así continuó con su trabajo. Fue coautor y contribuyó en gran cantidad de publicaciones en sus últimos años (más de 10 publicaciones están fechadas entre 2015 y 2019). En el 2017, durante el Simposio Internacional de Tortugas Marinas, se le rindió un emotivo homenaje, en donde se le destacó como el personaje que más influencia ha tenido en el estudio y los investigadores de las tortugas marinas. Esa fue la última reunión en la que participó.

Consolidar el Museo y establecer las bases para que los objetivos se cumplieran fueron las principales actividades que llenaron sus últimos años de existencia. Con el apoyo de su esposa Sibille, pudo completar su obra y poder verla en actividad. Estudiantes que realizaban pasantías en el Museo podían encontrarlo en alguna sala y tener todavía la oportunidad de conversar con él, recibir sus enseñanzas, escuchar sus anécdotas, pero sobre todo recibir el fruto del esfuerzo de un hombre visionario, la oportunidad de venir a estudiar a un sitio privilegiado. Peter falleció en Oviedo, el 25 de febrero del 2020, a los 76 años.

Es pertinente indicar que la revista African Sea Turtle Newsletter publicó un número especial como homenaje a Peter, en el cual, amigos y colaboradores escribimos nuestras experiencias y muchas anécdotas de su vida. Un total de 67 aportes fueron presentados, construyendo en conjunto el legado que Peter nos dejó, lo que a la vez refleja el impacto y la huella que marcó por los hábitats de las tortugas de todo el mundo.

\section{Agradecimientos}

Las historias compartidas por muchos amigos se convierten en parte de la historia que uno puede contar, por lo que quiero agradecer a todos los tortugólogos que en diferentes ocasiones me contaron sus anécdotas. Un especial agradecimiento a Jacques Fretey y a Manjula Tilawari, quienes coordinaron la edición de la revista homenaje en el African Sea Turtle Newsletter y me solicitaron escribir un artículo, lo que a su vez motivó realizar esta biografía. Jacques Fretey, además, facilitó la firma de Peter, las fotografías de F Bornin y las propias, con los respectivos permisos para utilizarlas.

\section{Referencias}

Bowen, B. W., Clark, A. M., Abreu-Grobois, F. A., Chaves, A., Reichart, H. A., \& Ferl, R. J. (1998). Global Phylogeography of the Ridley Sea Turtles (Lepidochelys spp.) as inferred from Mitochondrial DNA Sequences. Genetica, 101, 179-189. https://doi.org/10.1023/A:1018382415005

Ceriani, S. A. (2021). Peter's True Colors \& The Time I Almost Killed Peter C.H. Pritchard. [Special issue to Honor an Extraordinay Man Dr. Peter Pritchard]. African Sea Turtle Newsletter, 15, 32-33. https://static1.squarespace.com/static/5f4099de727c9b747f81d4b1/t/603d7e9f8f93856874bcb601/1614642930921/African+Sea+Turtle+Newsletter+\%2315.pdf

\begin{tabular}{|c|c|c|c|c|c|}
\hline 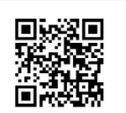 & (c) (1) (5) () & $\theta_{\text {AMEIENTIISS }}$ & $\frac{10 \%}{20}$ & $\frac{\text { UNA }}{\frac{\text { UNIVERSIDAD }}{\text { NACIONAL }}}$ & 266 \\
\hline
\end{tabular}




\section{Revista de CIENCIAS AMBIENTALES Tropical Journal of Environmental Sciences}
Revista de Ciencias Ambientales (Trop J Environ Sci) e-ISSN: 2215-3896 (Enero-Junio, 2022) . Vol 56(1): 259-267 DOI: https://doi.org/10.15359/rca.56-1.13 Open Access: www.revistas.una.ac.cr/ambientales e-mail: revista.ambientales@una.ac.cr Chaves A..

Chaves, A. (2021). Mi amigo Peter Pritchard. [Special issue to Honor an Extraordinary Man Dr. Peter Pritchard]. African Sea Turtle Newsletter, 15, 35-38. https://static1.squarespace.com/ static/5f4099de727c9b747f81d4b1/t/603d7e9f8f93856874bcb601/1614642930921/African+Sea+Turtle+Newsletter+\%2315.pdf

Fretey, J. (2021). Peter Charles Howard Pritchard: In Memoriam The Pope of the Turtles is Dead. [Special issue to Honor an Extraordinay Man Dr. Peter Pritchard]. African Sea Turtle Newsletter. 15, 7-17. https://static1.squarespace.com/static/5f4099de727c9b747f81d4b1/t/603d7e9f8f93856874bcb601/1614642930921/African+Sea+Turtle+Newsletter+\%2315.pdf

Pritchard, P. (1967). Living Turtles of the World. T.F.H. Publications

Pritchard, P. (1979). Encyclopedia of Turtles. T.F.H. Publications

Rhodin A. G. J. \& Thompson, S. (2020). Bibliography of Peter C.H. Pritchard. Chelonian Conservation and Biology, 19(1), 5-13. https://doi.org/10.2744/1071-8443.19.1.5

\begin{tabular}{|c|c|c|}
\hline 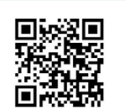 & (c) (1) (5) & 267 \\
\hline
\end{tabular}

